\title{
On Krasnoselskii's Cone Fixed Point Theorem
}

\author{
Man Kam Kwong ${ }^{1,2}$ \\ ${ }^{1}$ Department of Applied Mathematics, The Hong Kong Polytechnic University, Hunghom, Hong Kong \\ ${ }^{2}$ Department of Mathematics, Statistics, and Computer Science, University of Illinois, Chicago, \\ IL 60607-7045, USA
}

Correspondence should be addressed to Man Kam Kwong, mkkwong@uic.edu

Received 27 August 2007; Accepted 5 March 2008

Recommended by Jean Mawhin

In recent years, the Krasnoselskii fixed point theorem for cone maps and its many generalizations have been successfully applied to establish the existence of multiple solutions in the study of boundary value problems of various types. In the first part of this paper, we revisit the Krasnoselskii theorem, in a more topological perspective, and show that it can be deduced in an elementary way from the classical Brouwer-Schauder theorem. This viewpoint also leads to a topology-theoretic generalization of the theorem. In the second part of the paper, we extend the cone theorem in a different direction using the notion of retraction and show that a stronger form of the often cited Leggett-Williams theorem is a special case of this extension.

Copyright $\odot 2008$ Man Kam Kwong. This is an open access article distributed under the Creative Commons Attribution License, which permits unrestricted use, distribution, and reproduction in any medium, provided the original work is properly cited.

\section{Introduction}

The classical Brouwer-Schauder fixed point theorem is an undeniably important tool in the study of the existence of solutions to mathematical problems (e.g., see [1-3]). In recent years, another fixed point theorem due to Krasnoselskii [4,5] and its generalizations have been successfully applied to obtain existence results for multiple positive solutions of various types of boundary value problems, notably in the case of ordinary differential equations and their discrete versions. Krasnoselskii himself [5] has applied his result to study the existence of periodic solutions of periodic systems of ordinary differential equations. The main impetus for seeking new cone fixed point theorems is to apply them to obtain better criteria for the existence of solutions, for whatever problems the authors are currently interested in. The majority of known proofs of Krasnoselskii's theorem and its generalizations starts from first principles, mostly using topological index (degree) theory. Examples of direct proofs without using degree theory can be found, for example, in Potter [6] and Chaljub-Simon and Volkmann [7]. 
Krasnoselskii's theorem has two parts (to be described in Section 2). The first part, called the compressive form, bears resemblance to the Brouwer-Schauder theorem. In fact, in a recent paper [8], we show that the former is a special case of a generalized Brouwer-Schauder theorem. The second part, the expansive form, complements the compressive form. At first sight, it seems to call for a proof different from that of the Brouwer-Schauder theorem. In this paper, we are going to show that it follows from the compressive form almost trivially.

We believe that one of the reasons why the close relationship between Krasnoselskii's theorem and Brouwer-Schauder theorem has been overlooked is that the former is usually stated in the setting of a cone embedded in a Banach space with a given norm. In this setting, the norm functional plays a couple of important roles: in defining the region of points we are interested in, and in stating the properties of the images under the given map. When attempting to extend Krasnoselskii's theorem, one naturally focuses on finding similar functionals to replace the norm while still preserving these roles. On the other hand, the Brouwer-Schauder theorem is more topological in nature, being free from the concept of a metric. One can easily be misguided by this fact to think that the Brouwer-Schauder theorem is not adequate to deal with the metric aspects of cone maps.

The first goal of this paper is to point out that Krasnoselskii's theorem can indeed be interpreted in a nonmetric framework. The norm function is more of a convenience rather than a necessity. There are simpler ways to generalize the theorem without using functionals.

In Section 2, we first state a simplified version of Krasnoselskii's theorem and discuss several generalizations, especially the Krasnoselskii-Benjamin theorem. In Section 3, we discuss the topological nature of the simplified Krasnoselskii theorem and show that it is equivalent to a fixed point theorem for cylinder maps. We then show how the latter can be derived in an elementary way from the classical Brouwer-Schauder theorem. We present yet another proof of the expansive form of Krasnoselskii theorem. This proof makes it clear how we can formulate a generalized expansive cone result, which incidentally reads more like a Brouwer-type theorem than a cone theorem.

The second goal of this paper is to show that the boundary conditions in the Krasnoselskii theorem can be further generalized using the notion of retraction. The general result we present in Section 4 includes the Krasnoselskii-Benjamin theorem. Finally, in Section 5 we show how our general result implies the frequently quoted Leggett-Williams theorem as well as a result of Avery.

A discussion on applications of the new results derived here to boundary value problems is deferred to a future paper.

\section{Krasnoselskii's theorem}

The excellent expository article by Amann [9, Chapter 11] has a discussion and proof of the Krasnoselskii theorem, with the general boundary conditions (2.7) and (2.6). See also [5, 10].

Let $X$ be a (finite or infinite dimensional) Banach space with a given norm $\|\cdot\|$, and $K \subset X$ be a closed convex cone defined in the usual way, namely, that $K$ satisfies the following conditions:

(K1) If $x \in K$, then $\lambda x \in K$ for all real numbers $\lambda>0$,

(K2) If $x, y \in K$, then $x+y \in K$,

(K3) If both $x$ and $-x \in K$, then $x=0$,

(K4) $K$ is closed. 


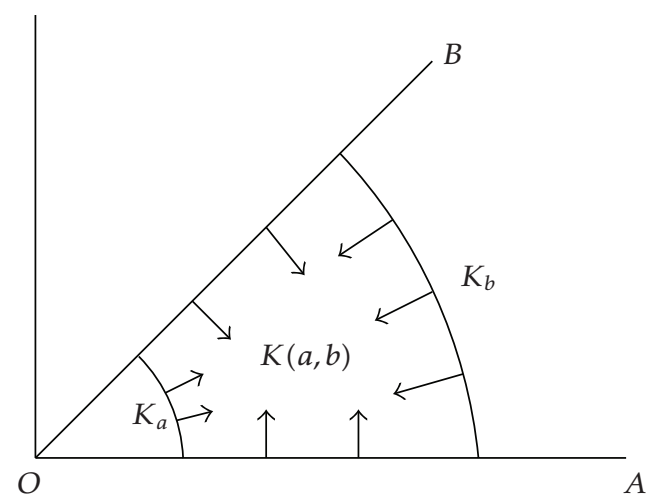

Figure 1: (Krasnoselskii's theorem in $R^{2}$ ) Compressive form.

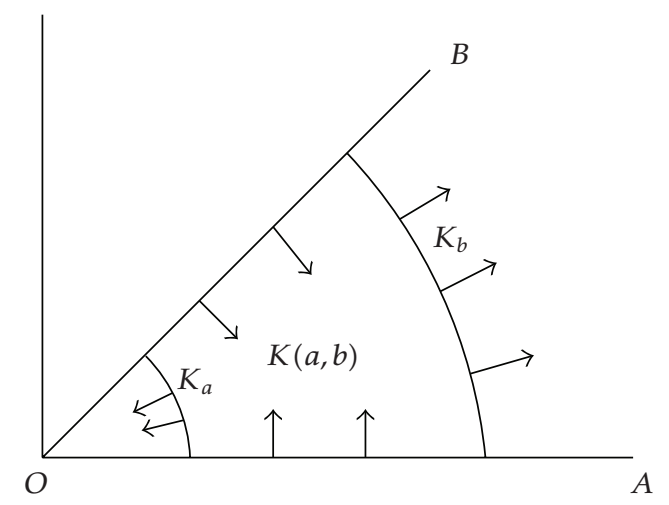

Figure 2: (Krasnoselskii's theorem in $R^{2}$ ) Expansive form.

For visualization, we can use the special case where $X$ is the three-dimensional space $R^{3}$ with the Euclidean norm, and $K$ is an infinite circular cone with its vertex at the origin, or, even more simply, use the case where $X$ is the two-dimensional plane $R^{2}$ and $K$ is the wedge-shaped region AOB in Figures 1 or 2.

A cone map on $K$ is a completely continuous map $T: K \rightarrow K$ (of $K$ into itself). When $X$ is finite dimensional, any continuous map is completely continuous. A point $x \in K$ is a fixed point of $T$ if $T(x)=x$.

Let $0<a<b$ be two given numbers. We are interested in conditions which guarantee that $T$ has a fixed point in the annular region $K(a, b)=\{x \in K: a \leq\|x\| \leq b\}$. Note that $K(a, b)$ is in general not convex, even though $K$ is. We denote by $K_{a}=\{x \in K:\|x\|=a\}$ and $K_{b}=$ $\{x \in K:\|x\|=b\}$ the inner and outer boundaries, respectively, of $K(a, b)$. We can extend the notation to define $K(0, a)$ and $K(b, \infty)$ in the obvious way. Theorem 2.1 is a simplified version of Krasnoselskii's original theorem. An illustration of this result in dimension 2 is depicted in Figures 1 and 2.

Theorem 2.1 (Krasnoselskii $1960[4])$. Let $K(a, b), T, K_{a}$, and $K_{b}$ be as defined above.

(1) (Compressive form) $T$ has a fixed point in $K(a, b)$ if

$$
\begin{aligned}
& \|T(x)\| \geq\|x\| \quad \forall x \in K_{a}, \\
& \|T(x)\| \leq\|x\| \quad \forall x \in K_{b} .
\end{aligned}
$$


(2) (Expansive form) $T$ has a fixed point in $K(a, b)$ if

$$
\begin{aligned}
& \|T(x)\| \leq\|x\| \quad \forall x \in K_{a}, \\
& \|T(x)\| \geq\|x\| \quad \forall x \in K_{b} .
\end{aligned}
$$

Note that the conditions (2.1)-(2.4) are imposed only on points on the two curved boundaries of $K(a, b)$. Interior points and points on the sides of the cone can be moved in any direction (as long as the image remains inside $K$ ). Also it is not stipulated that any particular image point $T(x)$ must lie inside $K(a, b)$.

The adjectives "compressive" and "expansive" in the names of the two forms of the theorem are conventional, and they are not meant to correctly describe the behavior of $T$ under all circumstances. For instance, in the "compressive" case, it may happen that the inner boundary $K_{a}$ is pushed by $T$ far beyond the outer boundary $K_{b}$, resulting in a much larger image $T(K(a, b))$ than $K(a, b)$.

When (2.1) (or (2.2)) holds, we say that $T$ is compressive on $K_{a}$ (or $K_{b}$ ) with respect to $K(a, b)$. The phrase "with respect to $K(a, b)$ " may be omitted if it is obvious from the context. If the inequality in (2.1) (or (2.2)) is strict, we say that $T$ is strictly compressive on $K_{a}$ (or $K_{b}$ ). Likewise when (2.3) (or (2.4)) holds, $T$ is expansive on $K_{a}$ (or $K_{b}$ ), and $T$ is strictly expansive if the inequality in (2.3) (or (2.4)) is strict.

The conventional technique to apply the cone fixed point theorem to obtain existence results for a boundary value problem is to rewrite the problem as an integral equation, usually via the use of Green's function. The Banach space is the space of continuous functions with an appropriate norm, and the positive cone is the set of continuous positive functions or some suitable subset of it. The integral operator is a completely continuous cone map and if one can find suitable constants $a$ and $b$ such that the hypotheses of the cone theorem are satisfied, then the annular region has a fixed point that is equivalent to a positive solution of the boundary value problem.

Many generalizations of Theorem 2.1 are known. The first direction of extension is to relax conditions (2.1)-(2.4). Krasnoselskii's original result is actually stated with weaker assumptions. In the compressive form, instead of (2.1) and (2.2), it is only required that

$$
\begin{array}{ll}
x-T(x) \notin K & \forall x \in K_{a}, \\
T(x)-x \notin K & \forall x \in K_{b} .
\end{array}
$$

This allows part (but not all) of the inner boundary $K_{a}$ to be pushed nearer the origin, and part of the outer boundary $K_{b}$ to be pushed away from the origin. Similar conditions are used by Krasnoselskii in place of (2.3) and (2.4) in the expansive form.

In [9], it is shown that these conditions can be further weakened. Amann attributes this result to Benjamin [11] (also established later independently by Nussbaum [12]). More precisely, conditions (2.1) and (2.2) can be replaced by

$$
\begin{gathered}
\exists p \in K \backslash 0, \quad \text { such that } x-T(x) \neq \lambda p \forall \lambda \geq 0, x \in K_{a}, \\
T(x) \neq \lambda x, \quad \text { for any } \lambda>1, x \in K_{b},
\end{gathered}
$$

and conditions (2.3) and (2.4) can be replaced by

$$
\begin{gathered}
T(x) \neq \lambda x, \quad \text { for any } \lambda>1, x \in K_{a}, \\
\exists p \in K \backslash 0, \quad \text { such that } x-T(x) \neq \lambda p \forall \lambda \geq 0, x \in K_{b} .
\end{gathered}
$$


In the literature (although not in [9]), condition (2.7) is called the Leray-Schauder condition. Schaefer [13] used it together with a retract argument and Schauder's fixed point theorem to prove the Leray-Schauder fixed point theorem. Petryshyn [14] has also used it to extend the Brouwer-Schauder theorem and applied it to obtain existence results of boundary value problems of partial differential equations. Some authors have thus referred to the above result as the Petryshyn-Krasnoselskii theorem. Following [9], we will refer to it as the KrasnoselskiiBenjamin theorem. A generalized Leray-Schauder condition is introduced in [8] to further extend Brouwer's theorem. In Section 4, we will show how this technique can also be used to extend the Krasnoselskii theorem.

Geometrically, (2.7) means that no point on $K_{b}$ is pushed by $T$ away from the origin "radially". In other words, pushing a point $x$ on $K_{b}$ above $K_{b}$ is allowed as long as the image point $T(x)$ is not collinear with $x$ and the origin. Geometrically, (2.6) means that no point on $K_{a}$ is pushed by $T$ towards the origin in a direction parallel to $p$; pushing it in the opposite direction away from the origin is allowed.

There is an apparent asymmetry in the pair of conditions (2.6) and (2.7), when compared to (2.1) and (2.2), or (2.5). An explanation will be given in Section 4 and the symmetry will be restored in our generalization of the Krasnoselskii-Benjamin result.

A second direction of extension is to look at regions more general than $K(a, b)$. A result due to Guo, see [10], replaces $K(a, b)$ in Theorem 2.1 by the more general region

$$
J=K \cap\left(\bar{\Omega}_{2} \backslash \Omega_{1}\right),
$$

where $\Omega_{1}$ and $\Omega_{2}$ are two bounded open sets in $X$ such that $0 \in \Omega_{1} \subset \bar{\Omega}_{1} \subset \Omega_{2}$, and $\bar{A}$ denotes the closure of a set $A$. We will also use $\partial A$ to denote the boundary of $A$. The conditions (2.1), (2.2) or (2.3), (2.4) are assumed to hold, but now for points on $K \cap \partial \Omega_{1}$ and $K \cap \partial \Omega_{2}$, instead of on $K_{a}$ and $K_{b}$, respectively. The hypotheses that $\Omega_{1}$ and $\Omega_{2}$ are open but otherwise arbitrarily means that we can apply the result to fairly general regions $J$. For instance, $J$ may contain holes. Most applications to differential equations, however, do not require such generalities. The new results in this paper are formulated for regions more general than $K(a, b)$, but not as general as in Guo's theorem.

The usual technique to obtain multiple solutions to a boundary value problem is to stack two or more annular regions together and apply the alternative forms of Krasnoselskii's theorem to each of the regions to get a fixed point. For example, take three positive numbers $0<a<b<c$, and define the corresponding regions $K(a, b)$ and $K(b, c)$. Let us assume that (2.1) and (2.2) hold for $K_{a}$ and $K_{b}$, and (2.4) holds for $K_{c}$ (replace $b$ in (2.4) by $c$ ). Then, there must be one fixed point in $K(a, b)$ and one fixed point in $K(b, c)$. There is a possibility that these two fixed points are one and the same. If so, it must lie on the common boundary $K_{b}$. In order to exclude this situation, we have to make the stronger assumption that $T$ maps $K_{b}$ strictly away from $K_{b}$, in other words, $T$ is strictly compressive on $K_{b}$ with respect to $K(a, b)$. In another example, if we assume that $T$ is strictly expansive on $K(a, b)$, and strictly compressive on $K(b, c)$, then we get at least three fixed points, one in each of $K(0, a), K(a, b)$, and $K(b, c)$.

Therefore, a third way to extend the cone theorem is to look for more general ways to construct such stacked-annulus structures. For instance, one may use the same inner and outer boundaries $K_{a}$ and $K_{c}$ as the example above, but replace $K_{b}$ by a set of points defined by some given continuous functional. The conditions (2.1)-(2.4) will, of course, have to be adjusted accordingly. Leggett and Williams [15] use a concave functional for this purpose. Avery [16] 
applies similar ideas to the boundaries $K_{a}$ and $K_{c}$, resulting in a five-functional theorem. In Section 4 , we will see that stronger forms of both of these results are corollaries of our general result.

\section{The topological nature of the fixed point property}

Let $B$ denote the closed unit ball in the Banach space $X$. The Brouwer-Schauder theorem is often stated in the following form:

Any completely continuous map of B into itself has a fixed point.

However, it is well known that this result can be applied to much more general sets. Let $A$ be a subset of $X$ that is topologically isomorphic (homeomorphic) to $B$. There exists a oneto-one topological map $F$, such that $F(B)=A$. If $S: A \rightarrow A$ is a completely continuous map, then the composite map $F^{-1} S F: B \rightarrow B$ is a completely continuous map, so that there is a fixed point, $F^{-1} S F(x)=x$. It follows that, $F(x)$ is a fixed point of $S$.

Suppose $K$ is a bounded closed subset of $X$ with the following star-shaped properties: there exists an interior point $O$, which has a neighborhood contained inside $K$, and for every point $A$ on the boundary of $K$, the line segment $O A$ is contained in the interior of $K$, except the end-point $A$. Then, it is obvious that $K$ is homeomorphic to the unit ball, via the topological map $F$ that scales every line $O A$ radially towards $O$ to be of unit length. Hence, the BrouwerSchauder theorem holds for $K$.

It is obvious that any bounded closed convex set with a nonempty interior satisfies the above star-shaped property. Therefore, the Brouwer-Schauder theorem holds for any bounded closed convex set with a nonempty interior. (In fact, it can be shown that this is true for any bounded closed convex set, but the weaker assertion suffices for our purpose in this paper.) In particular, this applies to the cylinder $C(0,1)$ that is used in Theorem 3.2 below. The cylinder $C(0,1)$ is defined as the cross product of the unit interval $[0,1]$ and the unit ball $B^{*}$ in the reduced space of codimension 1 , and is therefore convex.

An implication of the above observation is that the role played by the norm of the Banach space is not really that essential to the fixed point property (other than being used in the definition of bounded sets in $X$ ).

The same arguments can be applied to the Krasnoselskii theorem. We can topologically deform the cone $K$ and the annular region $K(a, b)$ in any way and still have a fixed point result. In the rest of this section we give two applications of this principle.

First let us deform $K(a, b)$ by moving every point on $K_{a}$ radially (and continuously) to a new point, while avoiding a neighborhood of the origin 0 . Likewise we can move every point on $K_{b}$ radially and continuously, while keeping it strictly "greater" than the corresponding point on the deformed $K_{a}$. The set $K(a, b)$ is now transformed to a new set $L$, which we can think of as a "finite segment" of the cone $K$ with continuous boundaries. Let us define this transformation more precisely and apply the above principle to a generalization of Theorem 2.1.

For every point $p$ on $K_{1}$, the ray (the half-infinite straight line) coming out from the origin towards $p$ intersects $L$ in a finite line segment $[\theta(p) p, \phi(p) p]$, where $0<\theta(p)<\phi(p)$ are real numbers that depend continuously on $p$. In addition, we assume that there exists a positive constant $\epsilon$ such that $\epsilon \leq \theta(p)$ for all $p$. $L$ is bounded from below by the inner boundary $L_{a}=\left\{\theta(p) p: p \in K_{1}\right\}$ and from above by the outer boundary $L_{b}=\left\{\phi(p) p: p \in K_{1}\right\}$, and on the side by the side of $K$. We keep the subscript $a$ and $b$ in the notation $L_{a}$ and $L_{b}$ to remind us 
that they are analogs of $K_{a}$ and $K_{b}$ in Theorem 2.1. They should have been named $L_{\theta}$ and $L_{\phi}$ instead.

If we take $\theta$ to be the constant function $\theta(p)=a$ and $\phi$ to be the constant function $\phi(p)=b$, then $L, L_{a}$, and $L_{b}$ coincide with $K(a, b), K_{a}$, and $K_{b}$ in the classical case, respectively.

Like $K(a, b), L$ is in general not convex, but both of them are "radially convex" in the sense that if two points in $L$ are collinear with the origin 0 , then the line segment joining the two points is contained in $L$.

We can extend the functions $\theta$ and $\phi$ to all $p \in K, p \neq 0$, by defining

$$
\theta(p)=\theta\left(\frac{p}{\|p\|}\right), \quad \phi(p)=\phi\left(\frac{p}{\|p\|}\right) .
$$

The geometric meaning of these functions are: a point $p$ lies "above" $K_{a}$ if and only if $\theta(p) \leq$ $\|p\|$; and it lies "below" $K_{b}$ if and only if $\|p\| \leq \phi(p)$.

Theorem 3.1. Let $L, L_{a}, L_{b}, \theta$, and $\phi$ be as described above, and let $T: L \rightarrow K$ be a completely continuous map.

(1) (Compressive form) $T$ has a fixed point in $L$ if

$$
\begin{array}{ll}
\|T(x)\| \geq \theta(T x) & \forall x \in L_{a}, \\
\|T(x)\| \leq \phi(T x) & \forall x \in L_{b} .
\end{array}
$$

(2) (Expansive form) $T$ has a fixed point in $L$ if

$$
\begin{aligned}
& \|T(x)\| \leq \theta(T x) \quad \forall x \in L_{a}, \\
& \|T(x)\| \geq \phi(T x) \quad \forall x \in L_{b} .
\end{aligned}
$$

Following the conventions used by some authors, we can also restate the result using some functionals. Let $\alpha: K \rightarrow[0, \infty)$ and $\beta: K \rightarrow[0, \infty)$ be two continuous functionals defined on the cone $K$, such that

$$
\alpha(x) \geq \beta(x) \quad \forall x \in K .
$$

We also require that they are strictly increasing in the radial direction, namely, that (the same holds for $\beta$ ):

$$
\alpha(x)>0 \quad \text { for } x \neq 0, \quad \alpha(\lambda x)>\alpha(x) \text { if } \lambda>1 .
$$

Let $0<a<b$ be two real numbers. Then, $L=\{x \in K: \alpha(x) \geq a, \beta(x) \leq b\}$ is a region as in Theorem 3.1 with boundaries $L_{a}=\{x \in K: \alpha(x)=a\}$ and $L_{b}=\{x \in K: \beta(x)=b\}$. Conditions (3.2)-(3.5) are then replaced by

$$
\begin{array}{ll}
\alpha(T(x)) \geq a & \forall x \in L_{a}, \\
\beta(T(x)) \leq b & \forall x \in L_{b} \\
\alpha(T(x)) \leq a & \forall x \in L_{a} \\
\beta(T(x)) \leq b & \forall x \in L_{b} .
\end{array}
$$


In our second application, we deform the annular region $K(a, b)$ into a cylinder

$$
C(0,1)=\left\{\left(t, x^{*}\right): 0 \leq t \leq 1, x^{*} \in B^{*}\right\},
$$

where $B^{*}$ is the unit ball in the reduced space of codimension 1 . To see this, first note that every point $x$ in $K(a, b)$ has the spherical coordinate $(\|x\|, x /\|x\|)$. Hence, $K(a, b)$ is isomorphic to $[a, b] \times K_{1}$. Here $K_{1}$ is the intersection of the unit sphere of the Banach space with the convex cone $K$. It is not the entire unit sphere, but rather, a proper "convex subset" of the unit sphere. It is "convex" in the sense that given any two points in $K_{1}$, the spherical "straight line" joining these two points is contained in $K_{1}$. We can then map $[a, b]$ linearly onto $[0,1]$ and deform $K_{1}$ to $B^{*}$. We can easily extend the isomorphism between $K(a, b)$ and $C(0,1)$ to an isomorphism between $K$ and the half-infinite cylinder

$$
C=\left\{\left(t, x^{*}\right):-1 \leq t, x^{*} \in B^{*}\right\} .
$$

Theorem 2.1 is thus equivalent to the next theorem, which is shown to follow from the classical Brouwer-Schauder theorem in an elementary way. We thus have a new proof of the Krasnoselskii theorem.

Theorem 3.2. Let $T: C(0,1) \rightarrow C$ be a completely continuous map, with the cylindrical coordinate representation:

$$
T(x)=(s, y), \quad-1 \leq s<\infty, y \in B^{*}
$$

(1) (Compressive form) $T$ has a fixed point in $C(0,1)$ if

$$
\begin{aligned}
& s \geq 0 \quad \forall x=\left(0, x^{*}\right), \\
& s \leq 1 \quad \forall x=\left(1, x^{*}\right) .
\end{aligned}
$$

(2) (Expansive form) $T$ has a fixed point in $C(0,1)$ if

$$
\begin{aligned}
& s \leq 0 \quad \forall x=\left(0, x^{*}\right), \\
& s \geq 1 \quad \forall x=\left(1, x^{*}\right) .
\end{aligned}
$$

Proof

Compressive form

As pointed out in [8], the compressive form is a special case of an extension of the BrouwerSchauder theorem, the so-called fixed point theorem with boundary conditions. Since the proof is not very long, it is repeated here.

Let $C_{0}$ and $C_{1}$ denote the bottom and top faces of the cylinder, respectively.

Recall that we have, at the beginning of this section, shown that $C(0,1)$ has the BrouwerSchauder fixed point property.

If $T$ maps $C(0,1)$ into itself, then the compressive form becomes just the BrouwerSchauder theorem. So suppose there are points $x \in C(0,1)$ that are mapped outside $C(0,1)$, 
that is, $T(x)=(s, y)$ with $s<0$ or $s>1$. Define

$$
T_{1}(x)=(\max (0, \min (1, s)), y) .
$$

The geometrical meaning of $T_{1}$ is: if $T(x)$ is in $C(0,1), T_{1}$ leaves it intact; if $T(x)$ is above $C_{1}$, then $T_{1}$ projects it vertically down to a point on $C_{1}$; and if $T(x)$ falls below $C_{0}$, then $T_{1}$ projects it vertically up to a point on $C_{0}$.

It is easy to see that $T_{1}$ is completely continuous and maps $C(0,1)$ into itself. So, by the Brouwer-Schauder theorem, $T_{1}$ has a fixed point $T_{1}\left(x_{0}\right)=x_{0}=\left(t_{0}, x_{0}^{*}\right)$. We claim that this must be a fixed point of the original map $T$. Suppose that $T\left(x_{0}\right)=\left(s_{0}, y_{0}\right)$.

There are three cases.

Case $1\left(0<t_{0}<1\right)$. In other words, $x_{0}$ is not on either $C_{0}$ or $C_{1}$. If $T\left(x_{0}\right)$ were above the upper face, $T_{1}$ would have pushed it down to lie on $C_{1}$. This contradicts the assumption that $x_{0}$ is a fixed point, because $x_{0}$ does not lie on $C_{1}$ while its image does. Likewise, $T\left(x_{0}\right)$ cannot be below $C_{0}$. Hence, $T\left(x_{0}\right)$ must be strictly between $C_{0}$ and $C_{1}$ and so $T\left(x_{0}\right)=T_{1}\left(x_{0}\right)=x_{0}$ and $x_{0}$ is a fixed point of the original map $T$.

Case $2\left(t_{0}=0\right)$. Now $x_{0}$ lies on $C_{0}$. By (3.12), $T\left(x_{0}\right)$ is on or above $C_{0}$. It cannot be above $C_{1}$, otherwise $T_{1}\left(x_{0}\right)$ will be on $C_{1}$ and $x_{0}$ cannot be a fixed point. Hence, $T\left(x_{0}\right)$ must be between $C_{0}$ and $C_{1}$ and so again $T\left(x_{0}\right)=T_{1}\left(x_{0}\right)=x_{0}$ and $x_{0}$ is a fixed point of the original map $T$.

Case $3\left(t_{0}=1\right)$. The proof is similar to Case 2 .

\section{Expansive form}

Without loss of generality we may assume that $T(x)$ has height $s \leq 2$, for all $x \in C(0,1)$. In the contrary case, we just redefine $T(x)=(\min (s, 2), y)$ and any fixed point of this new map is a fixed point of the original map.

Define a new map

$$
S(x)=S\left(t, x^{*}\right)=(2 t-s, y)
$$

It is easy to verify that $S$ is completely continuous. For a point $x$ on $C_{0}, t=0$, and $s \leq 0$, so that $2 t-s \geq 0$. In other words, $S$ maps $x$ above $C_{0}$. Likewise, for a point $x$ on $C_{1}, t=1$, and $s \geq 1$, so that $2 t-s \leq 1$. In other words, $S$ maps $x$ below $C_{1}$. The map $S$, therefore, satisfies the compressive form that has already been proved above. Hence, $S$ has a fixed point $x_{0}=\left(t_{0}, x_{0}^{*}\right)$. Thus, if $T\left(x_{0}\right)=\left(s_{0}, y_{0}\right)$, we have

$$
\left(t_{0}, x_{0}^{*}\right)=S\left(t_{0}, x_{0}^{*}\right)=\left(2 t_{0}-s_{0}, y_{0}\right)
$$

This implies that

$$
t_{0}=2 t_{0}-s_{0} \Longrightarrow t_{0}=s_{0}, \quad x_{0}^{*}=y_{0}
$$

Hence, $T\left(x_{0}\right)=\left(s_{0}, y_{0}\right)=\left(t_{0}, x_{0}^{*}\right)$ and $x_{0}$ is a fixed point of $T$.

The fact that the expansive form can be reduced to the compressive form opens up another direction of extension. However, we will not pursue this matter further in this paper, 
other than giving the following example. Let $A=\left\{x=\left(x_{i}\right)_{i=1, \ldots, n} \in R^{n}: 0 \leq x_{i} \leq 1\right\}$ be the unit cube in $R^{n}$. For each $i$, there are two faces $\left\{x \in A: x_{i}=0\right\}$ and $\left\{x \in A: x_{i}=1\right\}$ and there is an obvious way to define the concept of a compressive or expansive map on these faces in the $i$ th direction. Suppose that $T: A \rightarrow R^{n}$ is a continuous map that is either compressive or expansive in each $i$ th direction. Then $T$ has a fixed point. This idea has also been pursued in Precup [17], in which the product of $n$ annular regions is the analogue of the cube $A$.

In the special case when $A$ is a square, we have the interesting result: let $T: A \rightarrow R^{2}$ be a continuous function on a square A such that $T$ maps the upper edge to points above itself, the lower edge to points below itself, the left edge to points to its left, and the right edge to points to its right, then $T$ has a fixed point.

Let us give yet another proof of the expansive form of Theorem 3.2. This alternative proof is more complicated and less elegant than the one given above. However, it has the advantage of indicating how we can obtain a more general form of a multiple existence result for completely continuous maps.

\section{Alternative proof of the expansive form}

As before, we can assume that all $T(x)$ has height $s \leq 2$. We denote by $C(-1,0)=\left\{\left(t, x^{*}\right):-1 \leq\right.$ $\left.t \leq 0, x^{*} \in B^{*}\right\}$ and $C(1,2)=\left\{\left(t, x^{*}\right): 1 \leq t \leq 2, x^{*} \in B^{*}\right\}$ the two cylindrical regions below and above $C(0,1)$, respectively.

By assumption, the entire bottom face of the cylinder $C_{0}$ is mapped to a set $E_{0}$ inside $C(-1,0)$, and the entire top face $C_{1}$ is mapped to a set $E_{1}$ inside $C(1,2)$. We may even assume a little more, namely, that $E_{0}$ is strictly inside $C(-1,0)\left(E_{0}\right.$ does not intersect the boundary of $C(-1,0))$, and, likewise, that $E_{1}$ is strictly inside $C(1,2)$. If this is not the case, we can approximate $T$ by a sequence of completely continuous functions $T_{n}$, each having the desired property. Then each $T_{n}$ has a fixed point. The usual compactness argument then yields a convergence subsequence of these fixed points, whose limit can be shown to be a fixed point of $T$. The same approximation argument also allows us to assume that $T$ maps $C(0,1)$ strictly inside the cone $C$. It is easy to construct a continuous map $U: C(-1,2) \rightarrow C(-1,2)$ such that

(1) $U$ maps $C(-1,0)$ into itself and it shrinks the set $E_{0}$ to the single point $(-1 / 2,0)$, which is the center of the cylinder $C(-1,0)$;

(2) $U$ is the identity on $C(0,1)$;

(3) $U$ maps $C(1,2)$ into itself and it shrinks the set $E_{1}$ to the single point $(3 / 2,0)$.

It is also easy to verify that the composite map $U T: C(0,1) \rightarrow C(0,1)$ is completely continuous and any fixed point of $U T$ is a fixed point of $T$, and vice versa. But $U T$ has the nice property that it maps each of $C_{0}$ and $C_{1}$ to a single point.

Let us now extend $U T$ to a map $S: C(-1,2) \rightarrow C(-1,2)$, by requiring

$$
S(x)= \begin{cases}\left(-\frac{1}{2}, 0\right), & x \in C(-1,0), \\ \left(\frac{3}{2}, 0\right), & x \in C(1,2),\end{cases}
$$

$S$ maps $C(-1,2)$ strictly into $C(-1,2), C(-1,0)$ to the single point $(-1 / 2,0)$, and $C(1,2)$ to the single point $(3 / 2,0)$. 
It is well known that the existence of a fixed point for a map $S$ is implied by the assertion that the topological index of the map $V=(S-\mathrm{id})$ is nonzero at the point 0 , where id denotes the identity map. We know that $V$ has two fixed points, $(-1 / 2,0)$ and $(3 / 2,0)$. We claim that there must be at least one more.

By resorting to topological index theory, we see that the fact that $S$ maps $C(-1,2)$ strictly into itself implies that the index of $V$ at 0 is 1 or -1 , depending on how the index is defined, and the index is an odd number in any case. On the other hand, this index is the algebraic sum of the indices at all the fixed points. From the simple form of $V$ at the two known fixed points, we can see that the index at each of these points is either 1 or -1 . If there are no additional fixed points, then the algebraic sum of indices will be either 0 or \pm 2 (an even number in any case), which is a contradiction. This completes the proof.

It is now obvious how the same arguments can be used to obtain the following generalization of the expansive cone theorem. It does not read like a cone theorem, but it does imply the expansive Krasnoselskii theorem.

Theorem 3.3. Let $K_{1}, K_{2}, \ldots, K_{2 n}$ be a collection of $2 n$ nonoverlapping subsets of another subset $K$ of a Banach space. Assume that $K$ and $K_{i}$ are each isomorphic to the unit ball. Denote by $K_{i}^{o}$ the interior of each $K_{i}$, and

$$
L=K \backslash \bigcup_{i=1}^{2 n} K_{i}^{o} .
$$

Let $T: L \rightarrow K$ be a completely continuous map such that

$$
T\left(\partial K_{i}\right) \subset K_{i}, \quad \text { for } i=1, \ldots, 2 n,
$$

where $\partial K_{i}$ denotes the boundary of $K_{i}$. Then $T$ has a fixed point in $L$.

A simple example will be the unit ball $B$ with an even number of spherical holes inside it. Note that the result is false if there are only an odd number of holes.

\section{A cone theorem with generalized boundary conditions}

As mentioned in Section 2, we can extend Theorem 2.1 by discretely allowing parts of the boundaries $K_{a}$ or $K_{b}$ to be mapped to locations not allowed by (2.1) and (2.2), or by (2.3) and (2.4). The same can be said about Theorem 3.2. Let us explore this idea using the setting of the latter. We only discuss the compressive form in detail since the expansive form can be reduced to the compressive form by using the map $S$ (defined in the proof of Theorem 3.2) instead of $T$.

Suppose condition (3.12) is not true, namely, that part of the bottom face $C_{0}$ is mapped below itself. We want to be able to salvage the existence of a fixed point for $T$. By going through the proof of the compressive form of Theorem 3.2 carefully, we can see that the proofs for Cases 1 and 3 work exactly as before. Let us look at Case 2 . Recall that $x_{0}$ is a fixed point of $T_{1}$, and we need to show that $x_{0}$ is also a fixed point of $T$, or else there is a contradiction. If $T\left(x_{0}\right)$ happens to lie on or above $C_{0}$, the same proof still works. Therefore the only situation left to be dealt with is when $T\left(x_{0}\right)$ is below $C_{0}$. It is here that we need a condition to replace (3.12) with. The 
desired condition is to require that $y \neq x_{0}^{*}$, or equivalently, $T$ does not map $x_{0}$ directly under $x_{0}$. This condition rules out the possibility that $T_{1}$ pushes $T\left(x_{0}\right)$ back to its original position to get a fixed point. The proof of the existence of a fixed point for $T$ is thus complete.

In a similar way, if we know that $T$ does not map any point on the top face directly above itself, then the conclusion of the expansive form of Theorem 3.2 still holds. We have thus proved that the compressive form of Theorem 3.2 remains true if (3.12) and (3.13) are replaced by

$$
\begin{array}{ll}
\text { either } s \geq 0 \quad \text { or } \quad & y \neq x^{*} \forall x=\left(0, x^{*}\right), \\
\text { either } s \leq 1 \quad \text { or } \quad & y \neq x^{*} \forall x=\left(1, x^{*}\right) .
\end{array}
$$

If we translate these arguments back to the setting of the Krasnoselskii Theorem 2.1, (4.2) corresponds to requiring that $T$ does not move any point on $K_{b}$ radially away from the origin, and this is precisely the Leray-Schauder condition (2.7).

How about condition (4.1)? Why does it not translate into a similar Leray-Schauder-like condition for points on the inner boundary $K_{a}$ ? Why does there appear to be an asymmetry in the use of condition (2.7) for $K_{b}$ but condition (2.6) for $K_{a}$ ? It is true that conditions (4.1) and (4.2) are symmetric for the two boundaries of $C(0,1)$, but there is a little technical difficulty that precludes a perfect translation from the cylindrical framework back to the cone framework owing to the presence of the cone vertex (the origin $\mathrm{O}$ ). During the deformation of the cone to the cylinder, points very near to the vertex have to be moved in a different way than those points far away from the vertex. That is also why the cylindrical setting involves a half-infinite cylinder, from $t=-1$ to $t=\infty$. The asymmetry is inherent in the cone setting. As a consequence, there is no simple way to translate (4.1) into the Krasnoselskii setting if we have to deal with points near the plane $t=-1$, or equivalently points near the cone vertex.

Now that we know the trouble maker is the cone vertex, it is not hard to convince ourselves that as long as we know that, in the cone setting, the image of the inner boundary $K_{a}$ avoids a neighborhood of the origin, then an analogous Leray-Schauder condition will work for $K_{a}$ :

$$
T(x) \neq \lambda x, \quad \text { for any } 0 \leq \lambda<1, x \in K_{a} .
$$

In the special case when the Banach space $X$ is finite dimensional, (4.3) alone is sufficient, because the preliminary requirement that $T\left(K_{a}\right)$ is disjoint from a neighborhood of 0 follows from (4.3). To see this, first notice that (4.3) implies that 0 is not in the image $T\left(K_{a}\right)$. Being the image of a compact set $K_{a}$ (because $X$ is finite dimensional), $T\left(K_{a}\right)$ is also compact and so is closed. Thus, there must be a neighborhood of 0 that does not intersect $T\left(K_{a}\right)$.

Let us examine the proof used to establish (4.1) and (4.2) more closely to see what arguments can be further extended. A crucial step is the devising of a "retraction" map that pushes the part of the image $T(C(0,1))$ that lies outside $C(0,1)$ continuously onto $C_{0}$ or $C_{1}$. In our case, it is the vertical projection of the image point either up to $C_{0}$ or down to $C_{1}$. The analog in the cone setting is the radial projection of an image point either outwardly towards $K_{a}$ (if points in a neighborhood of 0 are not involved) or inwardly towards $K_{b}$.

As noticed in [8], in general, there exist a multitude of equally usable retractions for this purpose. Each retraction yields a generalized Leray-Schauder condition that can be used to extend the fixed point theorem (one merely repeats the above proof verbatim). This simple 
observation allows us to state a general result, given below as Theorems 4.1 and 4.2. They are formulated as an extension of Theorem 3.1 which includes the classical Theorem 2.1. Note that Theorem 3.3 can also be extended in a similar way, but we omit the details.

Going back to the case of trying to extend (2.1) and (2.4) for Theorem 2.1. If there is no way to avoid having image points near the origin, then the Leray-Schauder condition will not work. Instead we need to use conditions (2.6) and (2.9), which correspond to the retraction that pushes every point in $K(0, a)$ or $K(0, b)$ in the direction parallel to $p$ onto a point in $K_{a}$ or $K_{b}$. The Krasnoselskii-Benjamin theorem is thus a special case of the general result in this section.

To be more precise, a retraction of a topological space $Y$ onto a subset $Z \subset Y$ is a continuous map $f: Y \rightarrow Z$, such that its restriction to $Z$ is the identity map.

In addition to the notations of Theorem 3.1, we extend the analogy between $L$ and $K, L_{a}$ and $K_{a}$, and so forth, to define

$$
\begin{aligned}
L(0, a) & =\{x \in K:\|x\| \leq \theta(x)\}, \\
L(a, \infty) & =\{x \in K:\|x\| \geq \theta(x)\}, \\
L(0, b) & =\{x \in K:\|x\| \leq \phi(x)\}, \\
L(b, \infty) & =\{x \in K:\|x\| \geq \phi(x)\} .
\end{aligned}
$$

Given a completely continuous map $T: L \rightarrow K$, let $H_{a}$ be a subset of $L(0, a)$ that contains the union of $L(0, a) \cap T(L)$ and $L_{a}$, and let $H_{b}$ be a subset of $L(b, \infty)$ that contains the union of $L(b, \infty) \cap T(L)$ and $L_{b}$. For the expansive form, we need two similar sets. Let $\bar{G}_{a}$ be a subset of $L(a, \infty)$ that contains the union of $L(a, \infty) \cap T(L)$ and $L_{a}$, and let $\bar{G}_{b}$ be a subset of $L(0, b)$ that contains the union of $L(0, b) \cap T(L)$ and $L_{b}$.

Theorem 4.1 (compressive form). Suppose there exist two retractions $f_{a}: H_{a} \rightarrow L_{a}$ and $f_{b}: H_{b} \rightarrow$ $L_{b}$, and $T: L \rightarrow K$ satisfies

$$
\begin{array}{ll}
\text { either }\|T(x)\| \geq \theta(T x) \quad \text { or } \quad f_{a}(x) \neq x \forall x \in L_{a}, \\
\text { either }\|T(x)\| \leq \phi(T x) \quad \text { or } \quad f_{b}(x) \neq x \forall x \in L_{b} .
\end{array}
$$

Then, $T$ has a fixed point.

Theorem 4.2 (expansive form). Suppose there exist two retractions $g_{a}: \bar{G}_{a} \rightarrow L_{a}$ and $g_{b}: \bar{G}_{b} \rightarrow L_{b}$, and $T: L \rightarrow K$ satisfies

$$
\begin{aligned}
& \text { either }\|T(x)\| \leq \theta(T x) \quad \text { or } \quad g_{a}(x) \neq x \forall x \in L_{a} \text { ， } \\
& \text { either }\|T(x)\| \geq \phi(T x) \quad \text { or } \quad g_{b}(x) \neq x \forall x \in L_{b} \text {. }
\end{aligned}
$$

Then, $T$ has a fixed point.

For convenience, we call (4.5) and (4.6) the generalized compressive condition and (4.7) the generalized expansive condition.

Let us give a simple example of an application of Theorem 4.1. Figure 3 depicts a continuous map $T$ of a two-dimensional cone similar to the one shown in Figure 1 . $T$ is 


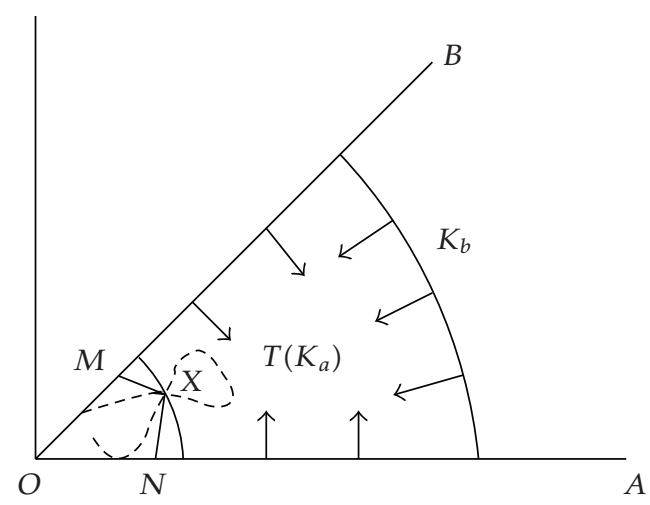

Figure 3: An example of the cone theorem with generalized boundary conditions.

compressive on the outer boundary $K_{b}$, but not on the inner boundary $K_{a}$. We require that $T\left(K_{a}\right)$ intersects $K_{a}$ at a single point $\bar{x}$, and that $\|T(\bar{x})\| \geq a$. Then, $T$ has a fixed point.

The dashed curve represents the image of $K_{a}, T\left(K_{a}\right)$. The point $X$ represents $\bar{x}$, where $T\left(K_{a}\right)$ intersects $K_{a}$. Note that as $x$ traverses the arc $K_{a}$ from one end to the other, the image $T(x)$ may cross $K_{a}$ multiple times (but every time at the same point $\bar{x}$ ). To prove the assertion, we have to construct the required retraction. Suppose we can draw two straight lines MX and NX as shown so that the part of the image $T\left(K_{a}\right)$ is contained in the quadrilateral OMXN. The desired retraction can be defined as follows: every point in OMXN is sent to $X$, every point above the line $\mathrm{MX}$ is pushed in a direction parallel to $\mathrm{MX}$ to a point on $K_{a}$, and every point at the right of NX is pushed in a direction parallel to NX to a point on $K_{a}$. Theorem 4.1 now gives us a fixed point. In general, if the curve $T\left(K_{a}\right)$ is somewhat tangential to $K_{a}$ at the point $X$, we may not be able to draw the lines MX and NX as shown. In such situations, we need to first deform $T\left(K_{a}\right)$ isomorphically until we are able to draw those lines. That should not be too hard to do.

The above example is a special case of the following more general situation. Let the arc $K_{a}$ be divided into three subarcs $x_{0} x_{1}, x_{1} x_{2}$, and $x_{2} x_{3} . x_{0}$ is where $K_{a}$ intersects the line OA; $x_{3}$ is where $K_{a}$ intersects $\mathrm{OB}$; and $x_{1}$ and $x_{2}$ are points on $K_{a}$. Suppose that $T\left(K_{a}\right)$ only crosses $K_{a}$ at points on the subarc $x_{1} x_{2}$, and $\|T x\| \geq\|x\|$ for all points on the subarc $x_{1} x_{2}$. Then, $T$ has a fixed point in $K(a, b)$.

Note that if the part of the image $T\left(K_{a}\right)$ that lies in the region OMXN touches the two sides of the cone as shown in Figure 3, then condition (2.6) is not satisfied. Therefore, Theorems 4.1 and 4.2 represent a true extension of the Krasnoselskii-Benjamin theorem.

Other examples of generalized Leray-Schauder conditions that are independent of the classical condition have been given in [8].

\section{The Leggett-Williams theorem as a special case}

A frequently cited extension of Krasnoselskii's theorem in the study of multiple solutions of boundary value problems is due to Leggett and Williams [15]. We will show that it is a special case of our general result in Section 4.

Let $\alpha: K \rightarrow[0, \infty)$ be a nonnegative continuous concave functional, and $\alpha(x) \leq\|x\|$, at least for those $x$ we are interested in. Let $0<a<b<d \leq c$ be given positive numbers. Define $K_{\alpha}(b, d)=\{x \in K: b \leq \alpha(x),\|x\| \leq d\}$. Assume that $T: K(0, c) \rightarrow K(0, c)$ is completely 


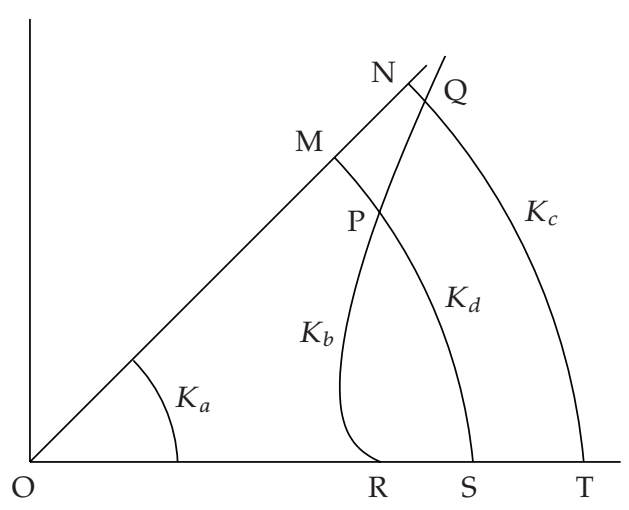

Figure 4: The Leggett-Williams theorem.

continuous and satisfies

(1) $\left\{x \in K_{\alpha}(b, d): \alpha(x)>b\right\} \neq \varnothing$ and $\alpha(T x)>b$, for $x \in K_{\alpha}(b, d)$,

(2) $\|T x\|<a$, for $\|x\| \leq a$, and

(3) $\alpha(T x)>b$, for $x \in K_{\alpha}(b, c)$ with $\|T x\|>d$.

Then, $T$ has at least three fixed points.

Recall the technique of stacked annulus discussed at the end of Section 2. In the LeggettWilliams result, the functional $\alpha$ is used in place of the norm in defining the middle boundary $K_{b}$. Leggett and Williams use the same $K_{a}$ and $K_{c}$ as in the classical case. It is obvious how the more general notions of $L, L_{a}$, and $L_{b}$ of Theorem 3.1 can also be exploited for this purpose.

It is useful to draw a picture to visualize the various sets involved. Figure 4 illustrates one possible situation. In the picture, the nonitalic letters from $\mathrm{M}$ to $\mathrm{T}$ are labels of points, while the italic $K_{a}, K_{b}, K_{c}$, and $K_{d}$ are names of curves. There are, of course, other possibilities. For instance, the curve QPR (or $K_{b}$ ) may intersect the curves $K_{d}$ and $K_{c}$ again near the bottom side of the cone. But Figure 4 suffices for our purposes.

Figure 4 is a flat representation of a higher-dimensional (even infinite dimensional) geometric object. So, we have to use a bit of imagination. When we see a curve, such as $K_{a}$, it is in fact a surface of codimension one, and a point of intersection, such as $\mathrm{P}$ is a surface of codimension two, and so on. For the sake of simplicity, in the discussion below, we stick to the two-dimensional terminologies of point and curve, and so on.

The curve $K_{b}$ represents the set of points $\{x \in K(0, c): \alpha(x)=b\}$. In general if the functional $\alpha$ is not strictly concave, this set may not be a "thin curve." The set $K_{\alpha}(b, d)$ is the area bounded by the curves PR, PS, and the straight line RS. The concavity of $\alpha$ and the convexity of the norm functional imply that this set is convex. The set $K_{\alpha}(b, c)$ is the area RPQTSR, which is also convex.

One obvious difference between Figure 4 and Figures 1 or 2 is the convexity of the curve $K_{b}$, because Leggett and Williams have replaced the norm functional, which is convex, by the concave functional $\alpha$ in the definition of $K_{\alpha}(b, d)$. As a consequence, the Leggett-Williams result is not a true extension of Krasnoselskii's theorem because it does not include the latter as a special case.

For the time being, let us ignore the curve $K_{d}$. The subset $K(0, c)$ is divided into three regions: $K(0, a), K_{\alpha}(b, c)$ (the area RPQTSR), and the rest. The circumstance here is reminiscent 
of that in the alternative proof of the expansive form of Theorem 3.2 (in which we have three cylinders $C(-1,0), C(1,2)$, and $C(0,1))$.

Condition 2 above implies that $T$ is strictly compressive on the first region, $K(0, a)$, and so it has a fixed point in the interior of $K(0, a)$. Notice that to arrive at this conclusion, we only need condition 2 to hold for all $\|x\|=a$, instead of for all $\|x\| \leq a$.

Now suppose we can show that $T$ is also strictly compressive on the region $K_{\alpha}(b, c)$. Then $K_{\alpha}(b, c)$ also has an interior fixed point. We can get a third fixed point by either using Theorem 3.3 or by using the fact that $T$ is expansive on the third region.

In general, under the hypotheses of the Leggett-Williams theorem, $T$ is not strictly compressive on $K_{\alpha}(b, c)$ in the simple sense, but we can show that it is strictly compressive in the generalized sense of Theorem 4.1. Hence, it becomes strictly expansive on the third region in the generalized sense. By applying Theorems 4.1 and 4.2, we will get two distinct fixed points, one in each of $K_{\alpha}(b, c)$ and the third region.

The region $K_{\alpha}(b, c)$ has two boundaries, the outer boundary is the part of $K_{c}$ between $\mathrm{Q}$ and T. The hypotheses that $T$ maps $K(0, c)$ into itself implies that $T$ is compressive on this outer boundary QT.

The inner boundary is the curve QPR, which is cut by the curve $K_{d}$ into two parts, the curve segments RP and PQ.

Condition 1 has two subconditions. The first implies that the set $K_{\alpha}(b, d)$ or RPSR has nonempty interior points. This subcondition is stronger than necessary; we need only to know that the curve segment RP is nonempty. The second subcondition means that $T$ pushes the area RPSR strictly to the right of the curve $K_{b}$ and this implies that $T$ is strictly compressive on $\mathrm{RP}$. It remains to show that $T$ is compressive in the general sense on the other part PQ of the boundary. This is where condition 3 comes in.

Condition 3 concerns $K_{a}(b, c)$, the entire region RPQTSR, but what we need to know to arrive at the desired result is only the information on the curve segment PQ. Let us restate condition 3: for $x$ on $\mathrm{PQ}$, either $\alpha(T x)>b$ or $\|T x\| \leq d$. Geometrically, this means that points on PW are mapped either to the right of $K_{b}$ or to the left of $K_{d}$. Those points that are mapped to the right of $K_{b}$ are being strictly compressed by $T$ and so we do not have to worry about them. It remains to show that those points that are mapped to the left of $K_{b}$ and $K_{d}$ (those image points that fall inside the region OMPR) are compressed by $T$ in the generalized sense.

To this end, we need to construct a retraction $f$ of the region OMNQPRO onto the curve QPR, such that the subregion OMPRO is collapsed onto PR. After this is done, take any $x$ on PQ. If $T(x)$ falls in OMPRO, then $f(T(x))$ lies on PR, and so $f(T(x)) \neq x$, and the generalized compressive condition (4.5) is satisfied.

There are many ways to construct the required retraction. For instance, we can take a point A inside the region RPSR and project every point in OMNQPRO radially onto QPR using A as the center. We have thus completed the proof of a stronger Leggett-Williams result, with conditions from 1 to 3 replaced by

(1') $K_{\alpha}(b, d) \neq \varnothing$ and $\alpha(T x)>b$, for $x \in K_{\alpha}(b, d) \cap K_{b}$,

(2') $\|T x\|<a$, for $\|x\|=a$, and

(3') $\alpha(T x)>b$, for $x \in K(c, d) \cap K_{b}$ with $\|T x\|>d$.

As a matter of fact, the way we treat $K_{b}$ in Figure 4 is reminiscent of how we treat $K_{a}$ in Figure 3. 
Since Leggett and Williams use the same boundaries $K_{a}$ and $K_{c}$ as in the classical case, that leaves some room for further generalization. In [16], Avery proves a five-functional theorem. His first idea is to define the curves $K_{a}, K_{c}$, and $K_{d}$ in a more general way using three distinct convex functionals instead of the norm functional. Then there is the same concave functional $\alpha$ as in Leggett-Williams. A fifth functional is used to define an additional curve that cuts $K_{a}$ into two parts, analogous to how $K_{d}$ cuts $K_{b}$ into two parts in the Leggett-Williams result (for this extension, condition 2 has to be extended to guarantee that $T$ when restricted to $K(0, a)$ is compressive on $K_{a}$ in the generalized sense). The geometrical configuration of the various sets is now a little more complicated than the Leggett-Williams setting. The curves $K_{c}$ and $K_{d}$ are still concave outwards, but since they are defined by two distinct functionals, they may not be disjoint as shown in Figure 4. In addition, there is a fifth curve that intersects $K_{a}$. Nevertheless, a careful repetition of the arguments in our proof of the LeggettWilliams theorem can be used to show that the five-functional theorem is likewise a corollary of Theorems 4.1 and 4.2 .

\section{Acknowledgment}

The author is thankful to the referee for many useful suggestions, among them the addition of references $[6,7,13,17]$.

\section{References}

[1] J. Cronin, Fixed Points and Topological Degree in Nonlinear Analysis, Mathematical Surveys, no. 11, American Mathematical Society, Providence, RI, USA, 1964.

[2] V. I. Istrăţescu, Fixed Point Theory. An Introduction, vol. 7 of Mathematics and Its Applications, D. Reidel, Dordrecht, The Netherlands, 1981.

[3] S. Tara, "Brouwer's fixed point theorem: methods of proof and applications," M.S. thesis, Simon Fraser University, Burnaby, BC, Canada, 2003.

[4] M. A. Krasnosel'skiǔ, "Fixed points of cone-compressing or cone-extending operators," Soviet Mathematics. Doklady, vol. 1, pp. 1285-1288, 1960.

[5] M. A. Krasnosel'skiı̌, The Operator of Translation Along the Trajectories of Differential Equations, American Mathematical Society, Providence, RI, USA, 1968.

[6] A. J. B. Potter, "A fixed point theorem for positive $k$-set contractions," Proceedings of the Edinburgh Mathematical Society II, vol. 19, pp. 93-102, 1974.

[7] A. Chaljub-Simon and P. Volkmann, "Existence of ground states with exponential decay for semilinear elliptic equations in $R^{n}$," Journal of Differential Equations, vol. 76, no. 2, pp. 374-390, 1988.

[8] M. K. Kwong, "On petryshyn's extension of Brouwer's fixed point theorem," to appear in Journal of Nonlinear Functional Analysis and Differential Equations.

[9] H. Amann, "Fixed point equations and nonlinear eigenvalue problems in ordered Banach spaces," SIAM Review, vol. 18, no. 4, pp. 620-709, 1976.

[10] D. Guo and V. Lakshmikantham, Nonlinear Problems in Abstract Cones, vol. 5 of Notes and Reports in Mathematics in Science and Engineering, Academic Press, San Diego, Calif, USA, 1988.

[11] T. B. Benjamin, "A unified theory of conjugate flows," Philosophical Transactions of the Royal Society of London. Series A, vol. 269, no. 1201, pp. 587-643, 1971.

[12] R. D. Nussbaum, "Periodic solutions of some nonlinear, autonomous functional differential equations. II," Journal of Differential Equations, vol. 14, no. 2, pp. 360-394, 1973.

[13] H. Schaefer, "Über die methode der a priori-Schranken," Mathematische Annalen, vol. 129, no. 1, pp. 415-416, 1955.

[14] W. V. Petryshyn, "On a fixed point theorem for nonlinear P-compact operators in Banach space," Bulletin of the American Mathematical Society, vol. 72, pp. 329-334, 1966. 
[15] R. W. Leggett and L. R. Williams, "Multiple positive fixed points of nonlinear operators on ordered Banach spaces," Indiana University Mathematics Journal, vol. 28, no. 4, pp. 673-688, 1979.

[16] R. Avery, "Existence of multiple positive solutions to a conjugate boundary value problem," Mathematical Sciences Research Hot-Line, vol. 2, no. 1, pp. 1-6, 1998.

[17] R. Precup, "A vector version of Krasnosel'skirı's fixed point theorem in cones and positive periodic solutions of nonlinear systems," Journal of Fixed Point Theory and Applications, vol. 2, no. 1, pp. 141-151, 2007. 